\title{
Variants in KIF1A gene in dominant and sporadic forms of hereditary spastic paraparesis
}

\author{
Andrea Citterio ${ }^{1} \cdot$ Alessia Arnoldi $^{1} \cdot$ Elena Panzeri $^{1} \cdot$ Luciano Merlini $^{2}$. \\ Maria Grazia D'Angelo ${ }^{3}$. Olimpia Musumeci ${ }^{4} \cdot$ Antonio Toscano $^{4}$. \\ Alice Bondi ${ }^{5} \cdot$ Andrea Martinuzzi $^{6} \cdot$ Nereo Bresolin $^{1,7} \cdot$ Maria Teresa Bassi $^{1}$
}

\begin{abstract}
KIF1A gene encodes the kinesin 1a protein, an axonal motor protein working in cargo transport along neurites. Variants in KIF1A were identified in different forms of neurodegenerative diseases with dominant and recessive inheritance. Homozygous recessive mutations were found in the hereditary sensory and autonomic neuropathy type 2, HSAN2 and in a recessive subtype of hereditary spastic paraparesis, SPG30. De novo heterozygous dominant variants were found both in a dominant form of SPG30 (AD-SPG30) with one single family reported and in patients with different forms of progressive
\end{abstract}

\& Maria Teresa Bassi

mariateresa.bassi@bp.lnf.it;

mariateresa.bassi@lanostrafamiglia.it

1 Laboratory of Molecular Biology, Scientific Institute IRCCS E. Medea, Via D. L. Monza 20, 23842 Bosisio Parini, Lecco, Italy

2 SC Laboratory of Musculoskeletal Cell Biology, Istituto Ortopedico Rizzoli, IRCCS, Bologna, Italy

3 Neuromuscular Disorders Unit, Scientific Institute IRCCS E. Medea, 23842 Bosisio Parini, Lecco, Italy

4 Department of Neurosciences, University of Messina, Messina, Italy

5 Department of Rizzoli-Sicilia, Istituto Ortopedico Rizzoli, 90011 Palermo, Italy

6 Conegliano Research Center, Scientific Institute IRCCS E. Medea, Conegliano, Italy

7 Neurology Unit, IRCCS Ca' Granda, Ospedale Maggiore Policlinico Foundation, Dino Ferrari Centre, Department of Physiopathology and Transplantation, Universita' di Milano, Milan, Italy neurodegenerative diseases. We report the results of a genetic screening of 192 HSP patients, with the identification of four heterozygous variants in KIF1A in four cases, two of whom with family history for the disease. Three of the four variants fall within the motor domain, a frequent target for variants related to the AD-SPG30 subtype. The fourth variant falls downstream the motor domain in a region lacking any functional domain. The KIF1A-related patients show clinical pictures overlapping the known AD-SPG30 phenotype including pure and complicated forms with few differences. Of note, one of the families, originating from the Sicily island, carries the same variant p.S69L detected in the first AD-SPG30 family of Finnish origin reported; differently from the first one, the latter family shows a wide intra-familial phenotype variability. Overall, these data reveal a very low frequency of the AD-SPG30 subtype while confirming the presence of amino acid residues in the motor domain representing preferential targets for mutations, thereby supporting their functional relevance in kinesin 1a activity.

Keywords NGS-targeted resequencing Spastic paraparesis _ KIF1A _ Dominant inheritance

\section{Introduction}

The KIF1A gene encodes a neuron-specific family 1 kinesin protein termed kinesin 1a, responsible for fast anterograde transport of synaptic vesicle precursors along axons [1, 2]. Both recessive and dominant variants in KIF1A gene were reported associated with a wide range of phenotypes with a different degree of severity. Homozygous recessive mutations in KIF1A were first described in patients affected by a severe form of a neurodegenerative disorder known as 
hereditary sensory and autonomic neuropathy type 2 (HSAN2) [3] and in three consanguineous families with an autosomal recessive form of hereditary spastic paraparesis termed SPG30 [4, 5]. De novo heterozygous dominant variants were found in a wide range of phenotypes as well. A specific mutation p.T99M was found in patients with intellectual disability (ID), spasticity, and axial hypotonia [6] and in another patient with a more severe phenotype including optic atrophy, growth failure, and progressive cerebellar atrophy, in addition to ID and spasticity [7]. A partly overlapping phenotype such as a progressive encephalopathy with brain atrophy was recently found associated with de novo KIF1A mutations [8]. Several de novo mutations and variants were found in patients classified either as pure or complicated HSP [9] and in one family with a dominant segregation pattern of pure HSP [10]. In all cases, the age range of disease onset is from 1 to 30 years. In the complicated phenotypes, the most common features accompanying spasticity are axonal neuropathy and brain cerebellar atrophy at the MRI [9, 10]. These authors suggested classifying both the recessive (AR) and the dominant (AD) forms of HSP associated with KIF1A variants as SPG30 [10]. The KIF1A variants associated with dominant and recessive SPG30 subtypes and with the progressive encephalopathy with brain atrophy are clustered in the motor domain of kinesin 1A, which is located in the $\mathrm{N}$ terminus of the protein (aa 1-365). It contains the key sequences for ATP and microtubules binding which are necessary for kinesin 1a movement along neurites. Additional functional domains are recognized downstream of the motor domain such as the fork-head domain (FHA, aa 516-572) with the neck coil, CC1-FHACC2-CC3, and a liprin alfa-binding and pleckstrin domain (PH, aa 1676-1774) [11]. The homozygous mutations in ARSPG30 affect the distal part of the motor domain [4, 5] while the mutations associated with HSAN2 are located just downstream the motor domain of the protein [3].

Here we report the results of a screening of a large series of HSP patients for KIF1A mutations, with the identification of four KIF1A variants in four index cases, two of whom showing family history for the disease. One of these two families carries the same dominant variant p.S69L detected in the first AD-SPG30 family previously reported [9] while showing a different phenotype. Three of the variants are located in the motor domain, while one falls within the middle portion of the protein upstream the $\mathrm{PH}$ domain.

\section{Materials and methods}

\section{Patients}

A series of 192 index patients with hereditary spastic paraplegia, HSP, were clinically evaluated and genetically analyzed. The study was approved by the Ethics Committee of the Scientific Institute E. Medea. Diagnosis of the HSP patients was based on the Harding criteria [12] for the definition of the clinical status. Seventy five patients had clinically pure HSP forms, while 117 showed a complicated phenotype characterized by a variable combination of axonal sensory-motor neuropathy, distal amyotrophy, and cognitive deficits in addition to spastic paraplegia. Age at disease onset ranged from 1 to 65 years of age. Sporadic occurrence of the diseases was observed in 120 patients; either a recessive or a dominant segregation pattern was observed in 42 and in 30 patients, respectively. Blood specimens were obtained from the probands, from all the available members of the families and from control subjects with informed consent.

\section{Sequencing}

For gene screening, a targeted next-generation sequencing (NGS) method was used that included 84 genes linked to HSP or overlapping diseases such as neuropathy, ataxia, and motor neuron diseases (Online Resource Table 1). Target enrichment and amplification were done with the HaloPlex kit (Agilent Technologies, Santa Clara, CA, USA) according to the manufacturer's instructions and sequenced on MiSeq (Illumina, San Diego, CA, USA) as described in detail in Supplementary Methods. Variant filtering steps and the relative results are reported in detail as Online Resource, Table 2. Sanger Sequencing was used to confirm all variants identified as previously described [13]. Accession no. of KIF1A cDNA and protein used for mutation nomenclature are NM_001244008.1 and NP_001230937.1, respectively.

Predictions of variants' pathogenicity were obtained using SIFT, PolyPhen-2, MutationTaster, MutPred, Panther, and PROVEAN software (Online Resource Fig. 1).

\section{Results}

\section{Genetic findings}

Search for KIF1A mutations through targeted NGS of 192 HSP patients led to the identification of four different heterozygous variants in the KIF1A gene, in two AD-HSP families and in two patients without family history for the diseases (Table 1; Fig. 1). No homozygous mutations/variants were found.

The variants, c.304G[A, p.G102S (novel), and c.499C TT, p.R167C (known [9]), were found in patients without family history of disease, P67809 and P116909, respectively. Parents were available for patient P67809 only; for him we were able to demonstrate the de novo 


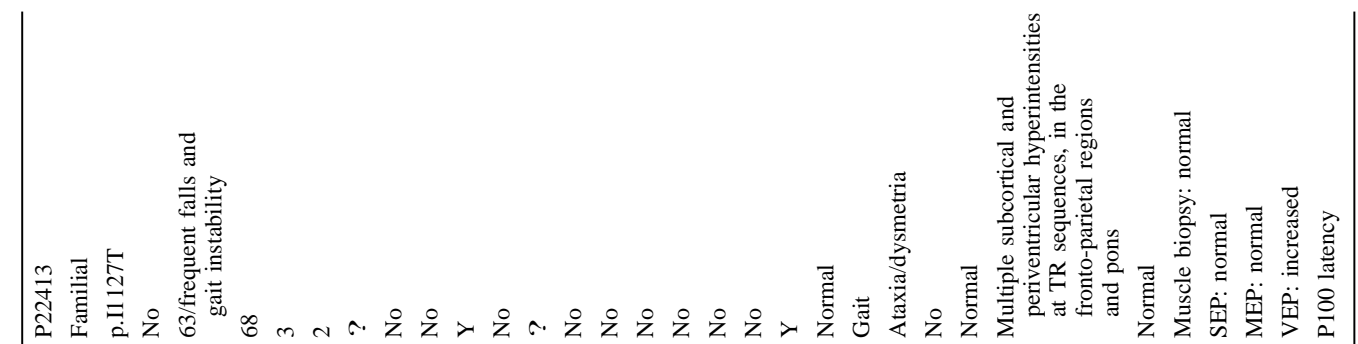

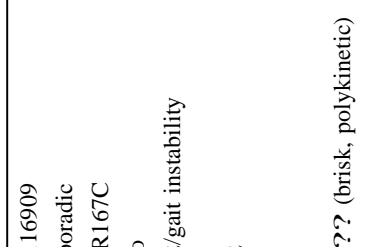

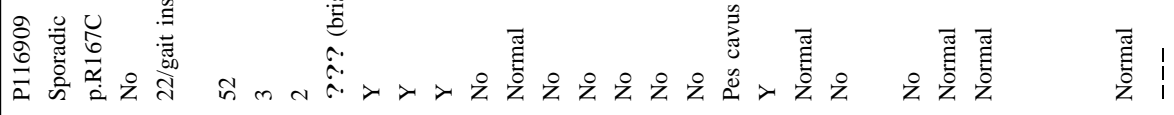

I

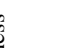

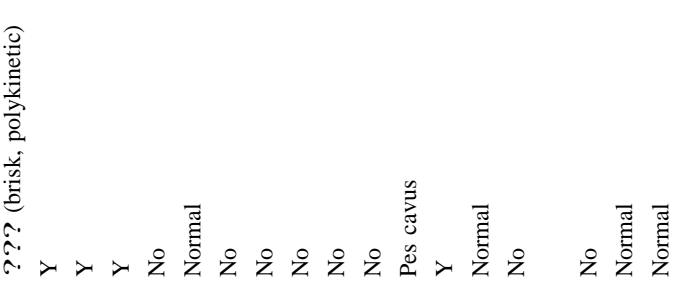

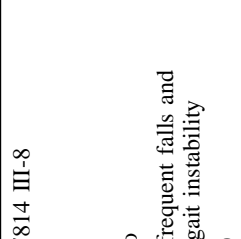

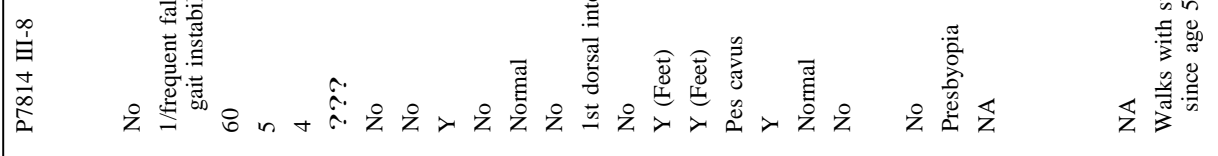

喜 至

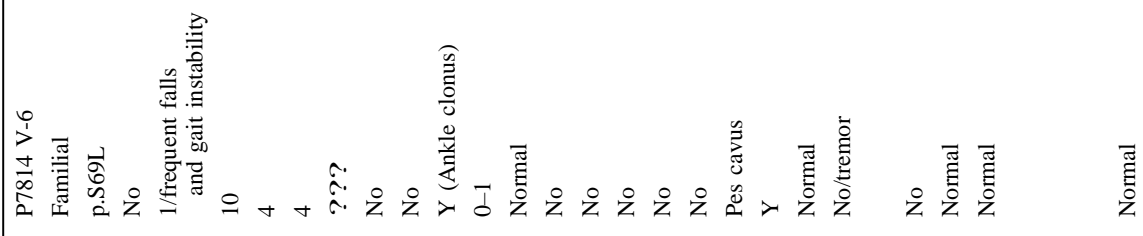

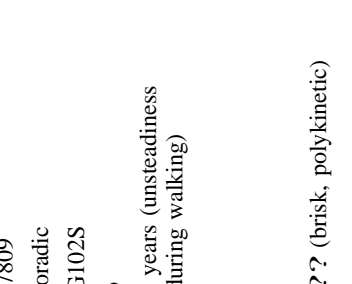

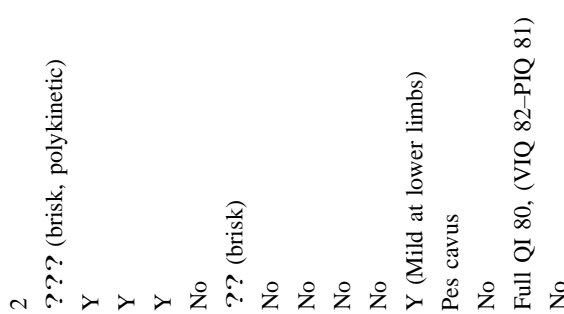

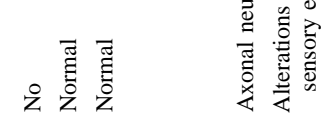

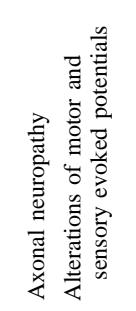

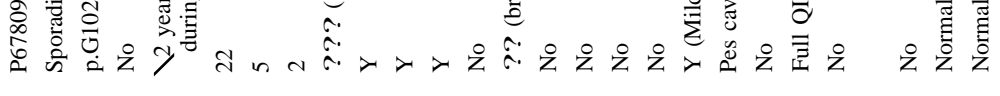

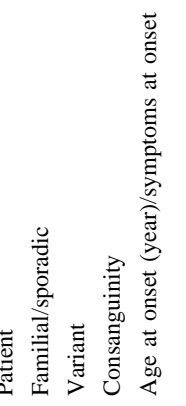

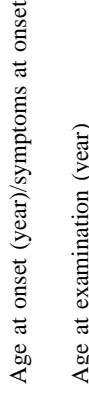
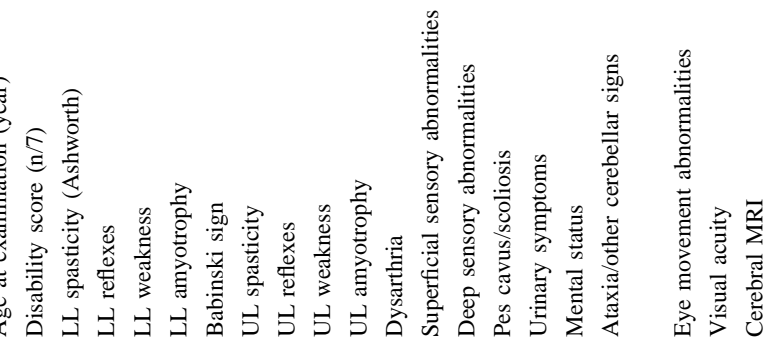

意言

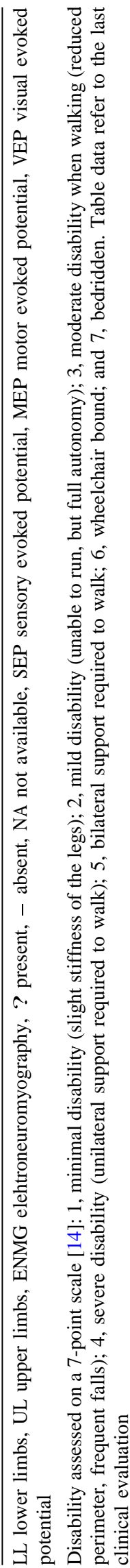


a

P7514

c.206C $>$ T; p.S69L

I

II

III

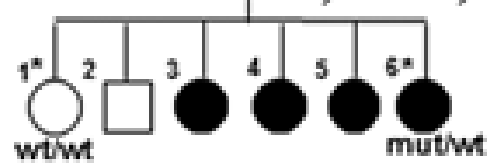

$\sqrt[4]{y^{5}}$

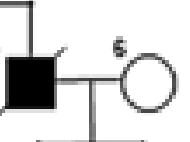

$7_{\text {mutwt }}^{8 *}$

IV

V
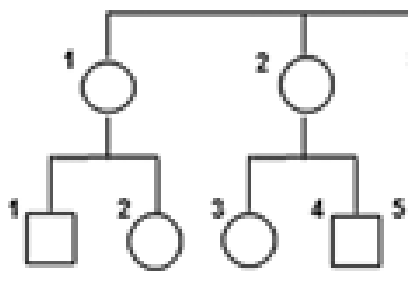

P22413

c. $3380 \mathrm{~T}>\mathrm{C}$ p.J1127T

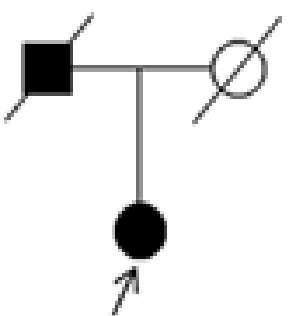

b

P7814

c. $206 \mathrm{C}>\mathrm{T}$ p. S69L

c.304G A P.G102S

P116909

P22413

C.499C $>$ T P.R167C

c.3380 $>$ C p.l112 $T T$

G C G TYG C A G

T G C C R G C

C C T T Y G C G T

T T C Y C C C
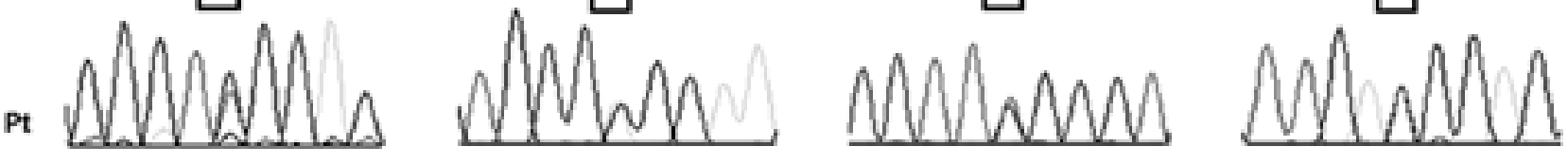

G C G T C C A

T G C C G C

C C T T C G G T

T T C T C C A C

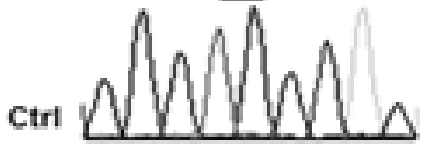
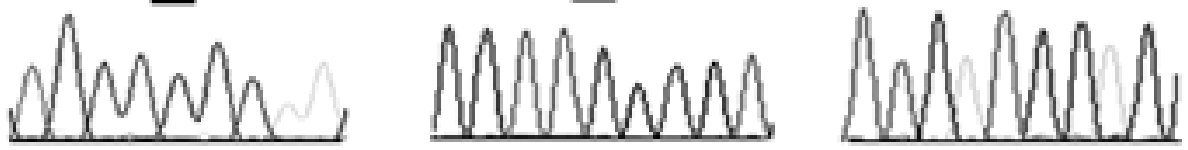

c

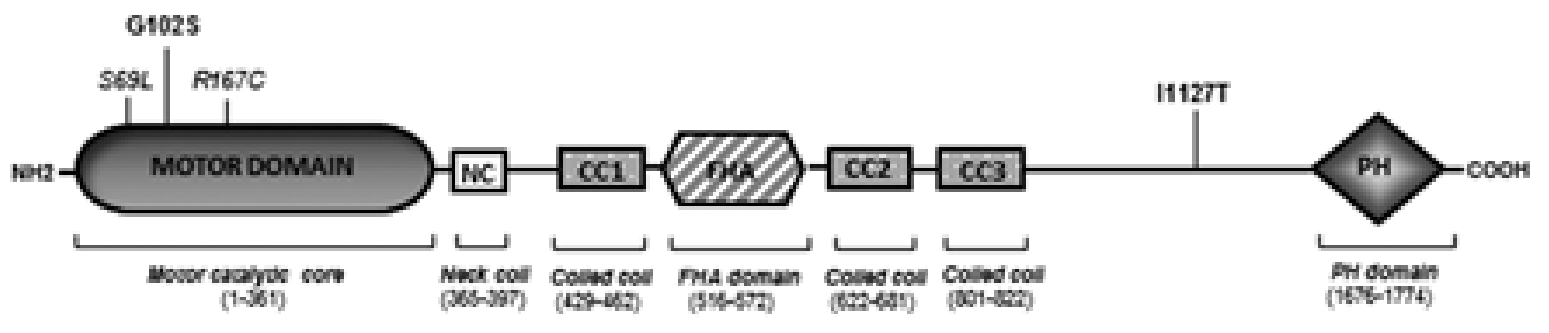

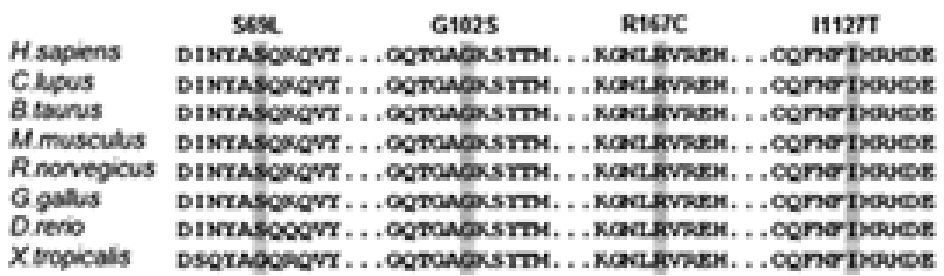


bFig. 1 a The Pedigree of the familial cases P7814 and P22413 carrying the p.S69L and p.I1127T variant, respectively. Black and white symbols indicate affected and healthy subjects, respectively. Arrow indicates the proband, asterisks indicated the subjects analyzed. b Electropherograms of all mutations identified in the study and control sequences. The variant nucleotides are boxed. c Schematic representation of KIF1A protein domains with the missense mutations described in this work in the upper part. Novel variants are indicated in bold. Partial alignment of the amino acid sequences encompassing the protein region carrying the variant residues. Accession no. of the aligned sequences: $H$. sapiens NP_001230937.1, C. lupus NP_001161945.1, B. taurus XP_00520 5116.1, M. musculus NP_032466.2 R. norvegicus XP_006245623.1, G. Gallus XP_003641781.1, D. rerio XP_005166002.1, and X. tropicalis XP_002933380.2

origin of the variant in the proband. The substitution p.G102S affects an amino acid residue (Gly102) involved in another change p.G102D in a previously reported patient [9].

In the dominant family P7814, the proband carries the dominant variant c.206C LT, p.S69L previously reported [10]. The change was inherited from the mother and was transmitted by the maternal grandfather (III-8) (Fig. 1); it was also detected in one of the four affected grandfather's cousins (III-6), who was available for both genetic and clinical evaluations. In the pedigree shown in Fig. 1, additional members are reported as affected based on clinical information obtained from the proband's parents and from the maternal grandfather.

A fourth variant c.3380T [C, p.I1127T was detected in patient P22413 with a family history for the disease. Unfortunately, only the proband of the family was available for genetic and clinical evaluations; therefore, segregation could not be checked.

Three out of the four variants are located in the motor domain; only p.I1127T falls within a region apparently lacking any functional domain, just upstream the $\mathrm{PH}$ domain. However, as shown in Fig. 1, all residues involved in the changes are highly evolutionary conserved. The novel variants were absent in 600 Italian control subjects; in dbSNP, 1000 genomes and EVS databases predictions for the novel variants indicate an impairment of protein functions in all the cases (Online Resource Fig. 1).

\section{Clinical findings}

Clinical and neuroradiological features of the patients carrying heterozygous variants in KIF1A are summarized in Table 1, while detailed clinical description is provided as Online Resource. They show either pure (P116909) or complicated forms of HSP. Mild intellectual disability or axonal neuropathy is typically associated with spasticity in the complicated HSP patients here described. Brain imaging was normal in all of them but one, P22413. Age of disease onset ranges from 1 to 63 years. The two familial cases here described originate both from Sicily Island and differ from the single AD-SPG30 family reported displaying a pure HSP with early onset [10]. In the family P7814, disease onset was still in early childhood and adolescence. However, a wide intra-familial phenotype variability is observed mostly in terms of diseases severity, ranging from an early-onset progressive spasticity associated with learning disability in the proband to a very mild disability mainly due to leg stiffness in the proband's mother, to a more severe phenotype in the maternal grandfather who displays also mild hand amyotrophy, superficial, and deep sensory abnormalities in the lower limbs in addition to spasticity. For family P22413, only the proband was available for clinical and genetic evaluations and thus reported in Table 1. Clinical data about the affected father (deceased) were reported by the proband. In this family, both the proband and her father manifested the first symptoms of gait instability and unsteadiness in their sixties. These symptoms rapidly evolved into lower limbs spasticity in 2-3 years. The father became wheelchair bound in about 10 years and died at age 87 . With exception of the late age of disease onset in patient P22413, the spectrum of clinical features displayed by these patients quite overlaps the clinical pictures previously described, associated with KIF1A-related dominant forms of HSP [9, $10]$.

\section{Discussion}

The genetic screening of a large series of HSP patients led to the identification of four heterozygous variants pS69L, p.G102S, p.R167C, and p.I1127T, in the KIF1A gene in four HSP cases, two sporadics and two with dominant inheritance. Two of the four variants are novel, p.G102S and p.I1127T. Overall, the clinical phenotypes displayed by these patients overlap the range of phenotypes previously described confirming a marked clinical variability associated with the AD-SPG30 subtype.

The three-generation family here described P7814, originating from the Sicily island, differs for several aspects from the single AD-SPG30 family so far reported with early-onset pure HSP [10]. This Sicilian family indeed presents a range of phenotypes recapitulating the phenotype variability shown by the sporadic cases so far reported. Indeed, the mildest form of the disease is observed in the proband's mother who had only a minimal disability with slight stiffness of the legs, pes cavus, positive Babinski sign, no intellectual disability, and no cerebellar signs. In the proband, the youngest affected of the family, spasticity was associated with psychomotor delay and learning disabilities. The most severe form of the disease 
was observed in the maternal grandfather who showed a mild hand amyotrophy, superficial, and deep sensory abnormalities in the lower limbs in addition to spasticity. In this case, no intellectual disability was found. Based on the family records, the affected cousins of the maternal grandfather showed a similar complicated phenotype with the same disease course. In spite of this, the Sicilian family shares the same dominant variant p.S69L previously detected in the Finnish family [10]. A possible explanation for such a phenotype variability associated with the same p.S69L variant might be derived from the structural modeling data available for the KIF1A motor domain [10]. Based on them, it appears that the S69 residue participates in the formation of the ATP-binding pocket, and the substitution of the serine residue for leucine could induce a conformational change likely breaking the interaction of the close residue Tyr67 with the ATP [10]. This would affect the stability of the ATP binding of KIF1A without altering the overall structure of the KIF1A ATP-binding pocket [10]. The effect on ATP-binding stability in turn might variably affect protein function and, even more, protein dimerization and dimer stability, thereby explaining the variability of the clinical outcome observed in the Sicilian family.

Based on the family records available and on the different ethnic origins, it is unlikely that the Sicilian and Finnish families share a common origin. In line with this hypothesis is the demonstration of the de novo origin of the p.S69L variant in the Finnish family. On a different level, the evidence of so many de novo mutations/variants detected in the KIF1A motor domain explains finding the same nucleotides and amino acids being affected several times in unrelated patients. Indeed, the $\mathbf{S} 69$ residue in the KIF1A motor domain is not the only example for that. Additional KIF1A amino acid residues are now emerging as frequent targets of substitutions such as T99 [6-9], R216 [8, 9], R167 ([9] and present study), E253, and R316 [8, 9], and even among the novel variants here detected, G102 has been found hit twice ([9] and present study). In fact, G102 undergoes to the p.G102S substitution herein described and to another presumed pathological change (p.G102D) previously reported [9]. The G102 residue lies in the conserved p-loop $\left(\mathrm{GX} 4 \mathrm{G}^{102} \mathrm{~K}[\mathrm{~T} / \mathrm{S}]\right)$ of the ATP-binding pocket of KIF1A motor domain. Based on structural modeling, the authors postulated that the previously reported substitution p.G102D disrupts the interaction with the phosphate region of ATP [9]. Although the change here described p.G102S, from Glycine to Serine, seems to be less dramatic in terms of charge and steric hindrance, it is likely that the key functional role of the p-loop has such strong sequence and structural requirements that even an apparently mild change cannot be tolerated. In line with that, apparently the two substitutions involving the same residue G102, p.G102D [9] and p.G102S (this study), are in both cases associated with a mild complicated phenotype characterized by ID and progressive spasticity in the first case, or by axonal neuropathy in addition to ID and spasticity in the latter. Concerning the p.R167C substitution, the postulated effect is to weaken the binding of the motor domain of KIF1A to the microtubules thereby affecting kinesin 1a movements along the neurites [9]. Indeed, the R167 residue is located in the loop 8 which binds the microtubules in an ATP hydrolysis-independent manner. In line with that, the patients carrying the p.R167C variant, ([9] and the one here described) both share a mild HSP phenotype.

The proband of the second family P22413 carries the novel KIF1A variant p.I1127T, which is located downstream the motor domain in a region apparently lacking any recognized functional domain. Although family history of patient P22413 indicates the presence of an affected deceased parent with overlapping clinical phenotype and age of disease onset, we do not have any chance to verify the segregation of the variant p.I1127T in the proband's father. We can only observe that the variant residue is highly evolutionary conserved and that all software used predict pathological/damaging effects for the substitution. The localization and type of the substitution may determine a mild functional effect of the variant identified which can fit with the late onset phenotype observed in the family. However, in the absence of any functional proof, at this stage, we favor the hypothesis the p.I1127T is a rare variant with an unknown significance rather than a mutation.

With regard to the novel variants detected in sporadic cases, although the range of clinical phenotype variability overlaps the one previously described for the AD-SPG30 patients [9], few known features cannot be observed in the patients here reported. For instance, cerebellar atrophy, reported to be typically associated with the AD-SPG30 subtype, cannot be found in the patients here described. In addition, EEG abnormalities or epilepsy, which were found in at least five patients previously reported [10], are not present in the patients of this study. Overall, the results of the screening of KIF1A gene in a large series of HSP patients indicate that KIF1A is not a frequent cause of HSP either pure or complicated forms. The lack of homozygous recessive mutations along with the few so far reported [4, 5] clearly suggests that recessive SPG30 is a very rare subtype of HSP. The identification of heterozygous dominant variants in 2 patients out of 120 sporadic and in 1 out of 30 dominant families (excluding the family with the rare variant of unknown significance p.I1127T), while confirming a low frequency of the dominant SPG30 subtype, still justifies the test of this gene in different forms of the HSP either with sporadic or familial occurrence regardless the age of disease onset. Additional studies on larger series 
of patients are needed to assess the correct frequency of the dominant form of the SPG30 subtype.

Acknowledgments The authors wish to thanks the patients and their families for participation to this work and all referring clinicians for collaboration. This work was supported by the Italian Ministry of health under the frame of E-Rare-2, the ERA-Net for Research on Rare Diseases" Grant NEUROLIPID and Grant No. 2015 and $5 \mathrm{xMille}$.

\section{Compliance with ethical standards}

Conflicts of interest On behalf of all authors, the corresponding author states that there is no conflict of interest.

Ethical standards All human studies have been approved by the appropriate ethics committee and have therefore been performed in accordance with the ethical standards laid down in 1964 Declaration of Helsinki and its later amendments. All persons gave their informed consent prior to their inclusion in the study. We have not included any details that might disclose the identity of the subjects in the study.

\section{References}

1. Lee JR, Shin H, Ko J, Choi J, Lee H, Kim E (2003) Characterization of the movement of the kinesin motor KIF1A in living cultured neurons. J Biol Chem 278:2624-2629. doi:10.1074/jbc. M211152200

2. Okada Y, Yamazaki H, Sekine-Aizawa Y, Hirokawa N (1995) The neuron-specific kinesin superfamily protein KIF1A is a unique monomeric motor for anterograde axonal transport of synaptic vesicle precursors. Cell 81:769-780. doi:10.1016/00928674(95)90538-3

3. Rivière JB, Ramalingam S, Lavastre V, Shekarabi M, Holbert S, Lafontaine J, Srour M, Merner N, Rochefort D, Hince P, Gaudet R, Mes-Masson AM, Baets J, Houlden H, Brais B, Nicholson GA, Van Esch H, Nafissi S, De Jonghe P, Reilly MM, Timmerman V, Dion PA, Rouleau GA (2011) KIF1A, an axonal transporter of synaptic vesicles, is mutated in hereditary sensory and autonomic neuropathy type 2. Am J Hum Genet 89:219-230. doi:10.1016/j. ajhg.2011.06.013

4. Erlich Y, Edvardson S, Hodges E, Zenvirt S, Thekkat P, Shaag A, Dor T, Hannon GJ, Elpeleg O (2011) Exome sequencing and disease-network analysis of a single family implicate a mutation in KIF1A in hereditary spastic paraparesis. Genome Res 21:658-664. doi:10.1101/gr.117143.110

5. Klebe S, Lossos A, Azzedine H, Mundwiller E, Sheffer R, Gaussen M, Marelli C, Nawara M, Carpentier W, Meyer V, Rastetter A, Martin E, Bouteiller D, Orlando L, Gyapay G, ElHachimi KH, Zimmerman B, Gamliel M, Misk A, Lerer I, Brice A, Durr A, Stevanin G (2012) KIF1A missense mutations in SPG30, an autosomal recessive spastic paraplegia: distinct phenotypes according to the nature of the mutations. Eur J Hum Genet 20:645-649. doi:10.1038/ejhg.2011.261

6. Hamdan FF, Gauthier J, Araki Y, Lin DT, Yoshizawa Y, Higashi K, Park AR, Spiegelman D, Dobrzeniecka S, Piton A, Tomitori H, Daoud H, Massicotte C, Henrion E, Diallo O, S2D Group, Shekarabi M, Marineau C, Shevell M, Maranda B, Mitchell G,
Nadeau A, D'Anjou G, Vanasse M, Srour M, Lafrenière RG, Drapeau P, Lacaille JC, Kim E, Lee JR, Igarashi K, Huganir RL, Rouleau GA, Michaud JL (2011) Excess of de novo deleterious mutations in genes associated with glutamatergic systems in nonsyndromic intellectual disability. Am J Hum Genet 88:306-316. doi:10.1016/j.ajhg.2011.02.001

7. Okamoto N, Miya F, Tsunoda T, Yanagihara K, Kato M, Saitoh S, Yamasaki M, Kanemura Y, Kosaki K (2014) KIF1A mutation in a patient with progressive neurodegeneration. J Hum Genet 59:639-641. doi:10.1038/jhg.2014.80

8. Esmaeeli Nieh S, Madou MR, Sirajuddin M, Fregeau B, McKnight D, Lexa K, Strober J, Spaeth C, Hallinan BE, Smaoui N, Pappas JG, Burrow TA, McDonald MT, Latibashvili M, Leshinsky-Silver E, Lev D, Blumkin L, Vale RD, Barkovich AJ, Sherr EH (2015) De novo mutations in KIF1A cause progressive encephalopathy and brain atrophy. Ann Clin Transl Neurol 2(6):623-635. doi:10.1002/acn3.198

9. Lee JR, Srour M, Kim D, Hamdan FF, Lim SH, Brunel-Guitton C, Décarie JC, Rossignol E, Mitchell GA, Schreiber A, Moran R. Van Haren K, Richardson R, Nicolai J, Oberndorff KM, Wagner JD, Boycott KM, Rahikkala E, Junna N, Tyynismaa H, Cuppen I, Verbeek NE, Stumpel CT, Willemsen MA, de Munnik SA, Rouleau GA, Kim E, Kamsteeg EJ, Kleefstra T, Michaud JL (2015) De novo mutations in the motor domain of KIF1A cause cognitive impairment, spastic paraparesis, axonal neuropathy, and cerebellar atrophy. Hum Mutat 36:69-78. doi:10.1002/humu. 22709

10. Ylikallio E, Kim D, Isohanni $P$, Auranen M, Kim E, Lönnqvist T, Tyynismaa H (2015) Dominant transmission of de novo KIF1A motor domain variant underlying pure spastic paraplegia. Eur J Hum Genet. doi:10.1038/ejhg.2014.297

11. Shin H, Wyszynski M, Huh KH, Valtschanoff JG, Lee JR, Ko J, Streuli M, Weinberg RJ, Sheng M, Kim E (2003) Association of the kinesin motor KIF1A with the multimodular protein liprinalpha. J Biol Chem 278:11393-11401. doi:10.1074/jbc. M211874200

12. Harding AE (1983) Classification of the hereditary ataxias and paraplegias. Lancet 1(8334):1151-1155. doi:10.1016/S01406736(83)92879-9

13. Citterio A, Arnoldi A, Panzeri E, D’Angelo MG, Filosto M, Dilena R, Arrigoni F, Castelli M, Maghini C, Germiniasi C, Menni F, Martinuzzi A, Bresolin N, Bassi MT (2014) Mutations in CYP2U1, DDHD2 and GBA2 genes are rare causes of complicated forms of hereditary spastic paraparesis. J Neurol 267:373-381. doi:10.1007/s00415-013-7206-6

14. Goizet C, Boukhris A, Mundwiller E, Tallaksen C, Forlani S, Toutain A, Carriere N, Paquis V, Depienne C, Durr A, Stevanin G, Brice A (2009) Complicated forms of autosomal dominant hereditary spastic paraplegia are frequent in SPG10. Hum Mutat 30:E376-E385. doi:10.1002/humu.20920

\section{Web Resources}

15. Genome database: http://browser.1000genomes.org/index.html

16. EVS: http://evs.gs.washington.edu/EVS/

17. NCBI dbSNP: http://www.ncbi.nlm.nih.gov/SNP/

18. SIFT and Provean: http://sift.jcvi.org/

19. MutPred: http://mutpred.mutdb.org/

20. Panther: http://www.pantherdb.org/tools/csnpScoreForm.jsp? 